
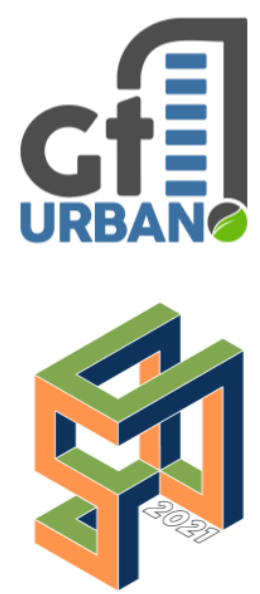

SINGEURB

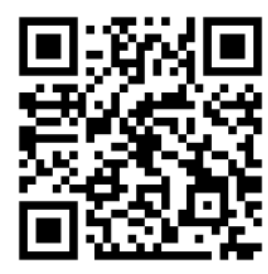

Como citar:

PORTO, Mattheus 1; DANILEVICZ,

Ângela 2; DE

MAGALHÃES,

Ruane 3. Atributos

Resilientes de

Sistemas de

Transporte Urbano.

In: III SIMPÓSIO

NACIONAL DE

GESTÃO E

ENGENHARIA

URBANA:

SINGEURB, 2021,

Maceió. Anais...

Porto Alegre:

ANTAC, 2021. p. 604-

611.

Disponível em:

https://eventos.antac. org.br/index.php/sin geurb/issue/view/14

\title{
Atributos resilientes de sistemas de transporte urbano
}

\section{Resilient attributes of urban transport systems}

Mattheus Souza Porto, Programa de Pós-Graduação em Engenharia Civil: Construção e Infraestrutura da Universidade Federal do Rio Grande do Sul, mattheusporto@gmail.com

Angela M. F. Danilevicz, Programa de Pós-Graduação em Engenharia Civil: Construção e Infraestrutura da Universidade Federal do Rio Grande doSul, angelamfd@ufrgs.br

Ruane Fernandes de Magalhães, Programa de Pós-Graduação em Engenharia de Produção da Universidade Federal do Rio Grande do Sul, ruane.magalhaes@gmail.com

\section{RESUMO}

A infraestrutura urbana de transportes, embora detenha centralidade no usufruto do direito de mobilidade pela população, é responsável por emissões de gases do efeito estufa, poluição do ar e congestionamentos. Ademais, a adequada provisão de serviços urbanos é fundamental para lidar com mudanças climáticas e crescimento populacional em cidades, as quais buscam adotar abordagens de planejamento considerando tanto sustentabilidade quanto resiliência para seu desenvolvimento sustentável. Ainda assim, poucos são os projetos de sistemas de transporte sustentáveis concebidos levando em conta seus impactos sobre a resiliência urbana. Neste contexto, o objetivo da dissertação de mestrado é a construção de um método de avaliação de resiliência urbana a ser aplicado em projetos de implementação e retrofit de sistemas sustentáveis de transporte em cidades. O método da pesquisa adota estratégia de Design Science Research e divide-se em 9 etapas compreendidas em coleta de informações, desenvolvimento e aplicação do método de avaliação. Atualmente, são realizadas atividades de revisão de literatura quanto à influência de sistemas de transporte na resiliência urbana. Ao decorrer da pesquisa, a principal contribuição esperada é o de guiar investimentos a serem realizados sobre a infraestrutura de transportes, considerando tanto a sustentabilidade quanto a resiliência na escala urbana.

Palavras-chave: Resiliência Urbana, Transportes, Sustentabilidade.

\begin{abstract}
Even though the urban transport infrastructure has a central role in providing the right of movement to population, it is also responsible for Greenhouse Gases emissions, air pollution and congestion. Moreover, the adequate provision of urban services is key to cope with climate change and population growth in cities, that are increasingly seeking urban planning approaches that consider both resilience and sustainability to accomplish sustainable development. In this sense, there are scarce projects of transport systems which consider its impacts over the city resilient capacity. The main objective of the master's dissertation is to build an urban resilience assessment method to be applied in deployment
\end{abstract}


and retrofit projects of urban transport systems. The research method adopts the Design Science Research strategy and is divided in nine different steps related to information gathering, development and application of the assessment method. At the present moment, literature review regarding the influence of transport systems over urban resilience is being performed. Along the research, the principal contribution of this work is to guide urban transportation investments considering both resilience and sustainability at the city level.

Keywords: Urban Resilience, Transportation, Sustainability.

\section{INTRODUÇÃO}

\subsection{Contextualização}

A concentração de pessoas vivendo em cidades está aumentando. Estima-se que cerca de dois terços da população mundial residirão em cidades em 2050 e este crescimento se dá majoritariamente em países em desenvolvimento (ONU, 2014). Apesar da pequena ocupação de terras, cidades são responsáveis por aproximadamente $70 \%$ do PIB global, $70 \%$ das emissões de gases estufa e $60 \%$ de todo consumo de energia no planeta (ONU-HABITAT, 2016). Os efeitos das mudanças climáticas e os esforços em prover serviços urbanos estão entre os maiores obstáculos relacionados ao crescimento das cidades, desafios que muitas cidades atualmente estão despreparadas para enfrentar (ONU-HABITAT, 2016). Ademais, a rápida urbanização induz dinâmicas sociais e transformações na demanda por infraestrutura. Quando isto ocorre desordenadamente, cidades tendem a se tornar mais vulneráveis a efeitos de choques agudos, como inundações e tremores de terra, e estresses crônicos provocados por mudanças climáticas (ROCKEFELLER \& ARUP, 2014).

\subsection{Problema de Pesquisa}

A eminência de circunstâncias desafiadoras leva as cidades a adotarem novas abordagens de planejamento. Dados os impactos ambientais que os seres humanos têm continuamente enfrentado atualmente, a consideração dos três pilares da sustentabilidade (ambiental, econômico e social) em projetos de infraestrutura se tornou uma necessidade para o desenvolvimento urbano. Além disso, cidades devem aprimorar suas capacidades de resiliência visando maior preparação e adaptação mesmo quando suscetíveis a grande variedade de mudanças. Com uma acelerada urbanização, superar os desafios requer soluções transformativas que busquem sustentabilidade, mas que cuidadosamente também considerem a resiliência urbana (ELMQVIST et al., 2019).

Mesmo assim, ainda existe uma lacuna em tornar a resiliência uma realidade na dimensão urbana. De acordo com Elmqvist et al. (2019), esforços para implementar planos e políticas transformativas em cidades para atingir metas de sustentabilidade geralmente não consideram a sustentabilidade e a resiliência como interconectadas, mas sim como aspirações de planejamento urbano independentes. Consequentemente, políticas e práticas correntes voltadas a sustentabilidade e resiliência, se não examinadas cautelosamente, podem gerar trade-offs entre si, em que maximizar eficiência, por exemplo, pode reduzir a resiliência urbana por não promover diversidade e redundância.

A partir de revisão de literatura, constata-se que, apesar da reconhecida importância da resiliência urbana em cidades, há pouco entendimento de como subsistemas de infraestrutura influenciam na construção da resiliência (GOLDBECK et al., 2019). Logicamente, este fato também se aplica a sistemas de transporte, os quais muitas vezes possuem projetos de implementação e de retrofit que promovem a sustentabilidade, mas sem considerar seus possíveis impactos sobre a resiliência urbana. 
Desta forma, o problema de pesquisa do presente trabalho é definido como o incompleto entendimento de como projetos de implementação e retrofit de sistemas sustentáveis de transporte promovem a construção da resiliência urbana.

\subsection{Questão e Objetivos de Pesquisa}

Dadas as circunstâncias envolvendo a lacuna do conhecimento e o problema de pesquisa, a questão de pesquisa do trabalho pode ser estabelecida da seguinte forma: Como projetos de implementação e retrofit de sistemas de transporte sustentáveis podem aprimorar a resiliência urbana?

Para responder à essa questão, o objetivo geral do trabalho é o de desenvolver um método de avaliação de resiliência urbana para ser aplicado em projetos de implementação e retrofit de sistemas de transporte em cidades.

Para auxiliar no atingimento do objetivo geral, outros três objetivos específicos são delineados:

a) Identificar os principais atributos de sistemas de transportes que mais influenciam na construção da resiliência urbana;

b) Caracterizar as relações existentes entre sistemas de transporte e a resiliência urbana;

c) Definir oportunidades de adaptação de sistemas de transporte sustentáveis, visando maiores níveis de resiliência urbana.

\section{REFERENCIAL TEÓRICO}

\subsection{Resiliência Urbana e Sustentabilidade}

Resiliência urbana é um parâmetro amplo e cada subsistema de infraestrutura, como energia e transportes, por exemplo, possui uma parcela de contribuição em sua formação (GOLDBECK et al., 2019). Devido às suas diversas possíveis interpretações, o presente trabalho considera resiliência urbana com a seguinte definição: A habilidade de um sistema urbano e de todas as suas redes sociotécnicas e socioecológicas em manterem ou de retomarem rapidamente funções fundamentais frente a distúrbios, de se adaptarem a mudanças e de amplificarem sua capacidade de adaptação futura (MEEROW et al., 2016).

Para enfrentar os desafios urbanos atuais, é essencial examinar a existência de interações entre os subsistemas de infraestrutura, mesmo que alguns destes, como transporte e energia, tenham sido inicialmente concebidos sem levar em conta suas interconexões (PANDIT et al., 2017). Dado o grau de incerteza do cenário urbano futuro (crescimento populacional, vulnerabilidade aos padrões climáticos pouco conhecidos e aumento de demandas sobre a infraestrutura), a construção de resiliência contra desastres inesperados requer uma abordagem holística (COLLIER et al., 2013). Ademais, a potencialização da resiliência vislumbrando a promoção de sustentabilidade é crucial para alcançar futuros estados desejados de desenvolvimento sustentável (TIMON, 2014). 


\subsection{Sistemas de Transporte em Cidades}

Serviços urbanos de transporte são os facilitadores do direito de mobilidade para moradores em uma cidade, já que um eficiente sistema de transporte urbano é o meio pelo qual a população usufrui de uma vasta quantidade de oportunidades econômicas. Apesar de sua inegável importância, o setor de transportes é um dos maiores responsáveis por emissões de gases estufa e por questões como mortes no trânsito, congestionamentos urbanos e isolamento de cidadãos com acesso precário a este serviço (ICLEI, 2019).

No contexto brasileiro, atrasos causados por congestionamento chegam a representar economicamente cerca de 10\% do PIB na cidade de São Paulo (ONU-Habitat, 2013). Adicionalmente, Carvalho (2011) declara que em grandes áreas urbanas brasileiras, cerca de $57 \%$ das emissões de dióxido de carbono são provenientes de carros, $27 \%$ provêm de transporte público e $12 \%$ do transporte de carga. Para encarar este cenário, sistemas de transporte sustentáveis devem progressivamente adotar, por exemplo, medidas de melhor acessibilidade ao transporte público, aprimorar eficiência energética e melhorar as condições para mobilidade não motorizada (ICLEI, 2019).

Além disso, sistemas de transporte urbano requerem expressivas quantidades de capital para serem concebidos. Em cidades localizadas nas Américas Latina e Caribenha, por exemplo, os setores de transportes representam o serviço público com as maiores necessidades de investimento (BONILLA; ZAPPAROLI, 2017).

\section{MÉTODO}

Dados o contexto e os objetivos do trabalho, julga-se a estratégia de Design Science Research como a mais adequada para atingir o objetivo geral. Com a estratégia definida, o delineamento do trabalho é estruturado em três grandes etapas de atividades (Compreensão, desenvolvimento e reflexão), sinteticamente apresentadas na Figura 1 e descritas com mais detalhes a seguir.

A fase de compreensão da pesquisa refere-se à parcela descritiva do estudo. Dividida em quatro atividades sequenciais, ela envolve o levantamento de informações relativas aos (1) atributos de sistemas de transportes que possivelmente influenciam na resiliência urbana, aos (2) requisitos de Instituições Financeiras Multilaterais (IFM) e bancos de desenvolvimento ao fomentarem sistemas sustentáveis de transporte (SST), à (3) combinação dos atributos identificados nas etapas anteriores e ao (4) entendimento da influência desses atributos na resiliência urbana.

Para a atividade (1), planeja-se uma revisão de literatura englobando publicações na temática de SST, preferencialmente realizadas entre os anos de 2011 e 2021. A seguir, a atividade de número (2) propõe um levantamento documental, para compreender o que é priorizado por instituições financeiras ao conceberem suporte para SST em países em desenvolvimento. Em seguida, na atividade (3), serão combinadas a lista preliminar de atributos de SST (1) e a lista de requisitos de IFM (2), com o intuito de identificar os principais atributos de SST que possivelmente influenciam na construção da resiliência, atingindo o objetivo específico $\mathrm{n}^{\mathrm{o}}$ 1. Para a atividade (4), busca-se entender mais profundamente as influências existentes entre os principais atributos de SST identificados em métricas e indicadores de resiliência existentes na literatura. Como resultado, espera-se atingir o objetivo específico $n^{\circ} 2$. 
Figura 1: Delineamento da pesquisa

\section{Delineamento da Pesquisa}

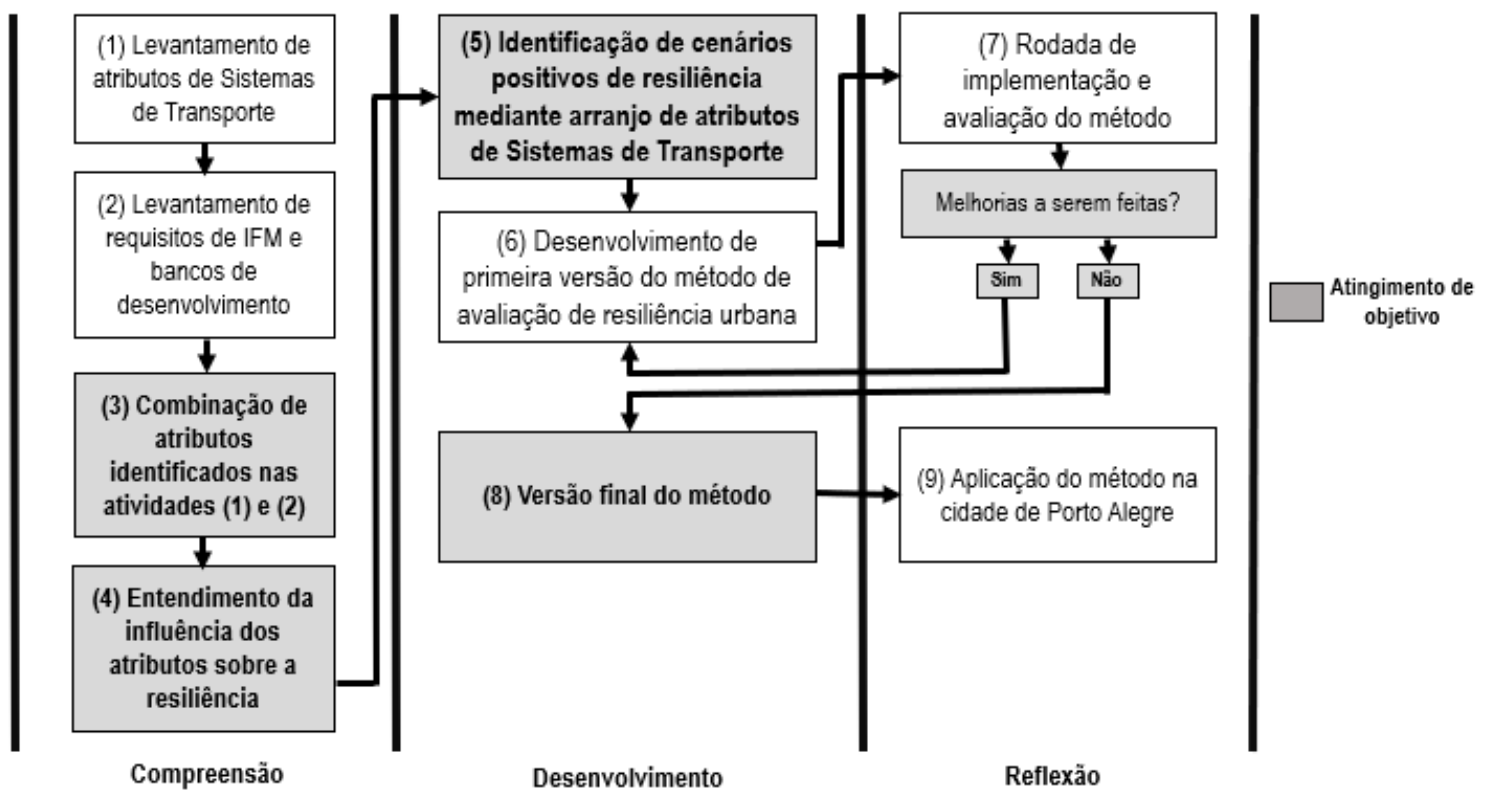

$\mathrm{Na}$ fase de Desenvolvimento, a pesquisa se volta ao âmbito prescritivo e maior é o enfoque dado às atividades de construção do método que avalia o impacto de projetos de implementação e retrofit de sistemas de transporte sobre a resiliência urbana. Na atividade (5), estipulam-se cenários de resiliência urbana mediante diferentes arranjos dos atributos de SST identificados na fase de compreensão. Para isso, será realizada uma análise comparativa de como atingir cenários positivos de resiliência, mediante a adoção de diferentes intervenções de SST. Assim, são identificadas oportunidades de adaptação de sistemas de transporte urbano que apoiem a construção da resiliência, atingindo o objetivo específico no 3 .

Seguindo à atividade (6), inicia-se o desenvolvimento da primeira versão do método de avaliação de resiliência urbana. Assim, toma-se como base o modelo conceitual proposto por Mendizabal et al. (2018), cujo propósito é o de orientar cidades na transição para contextos de maiores níveis de resiliência e sustentabilidade (Figura Erro! Fonte de referência não encontrada.2). Os autores do modelo declaram que este pode ser aplicado tanto no aprimoramento da resiliência, em um cenário geral da cidade, ou em uma parcela de resiliência urbana, abrangida por apenas um subsistema de infraestrutura em específico, a exemplo do setor de transportes. 
Figura 2 - Modelo conceitual proposto por Mendizabal et al. (2018)

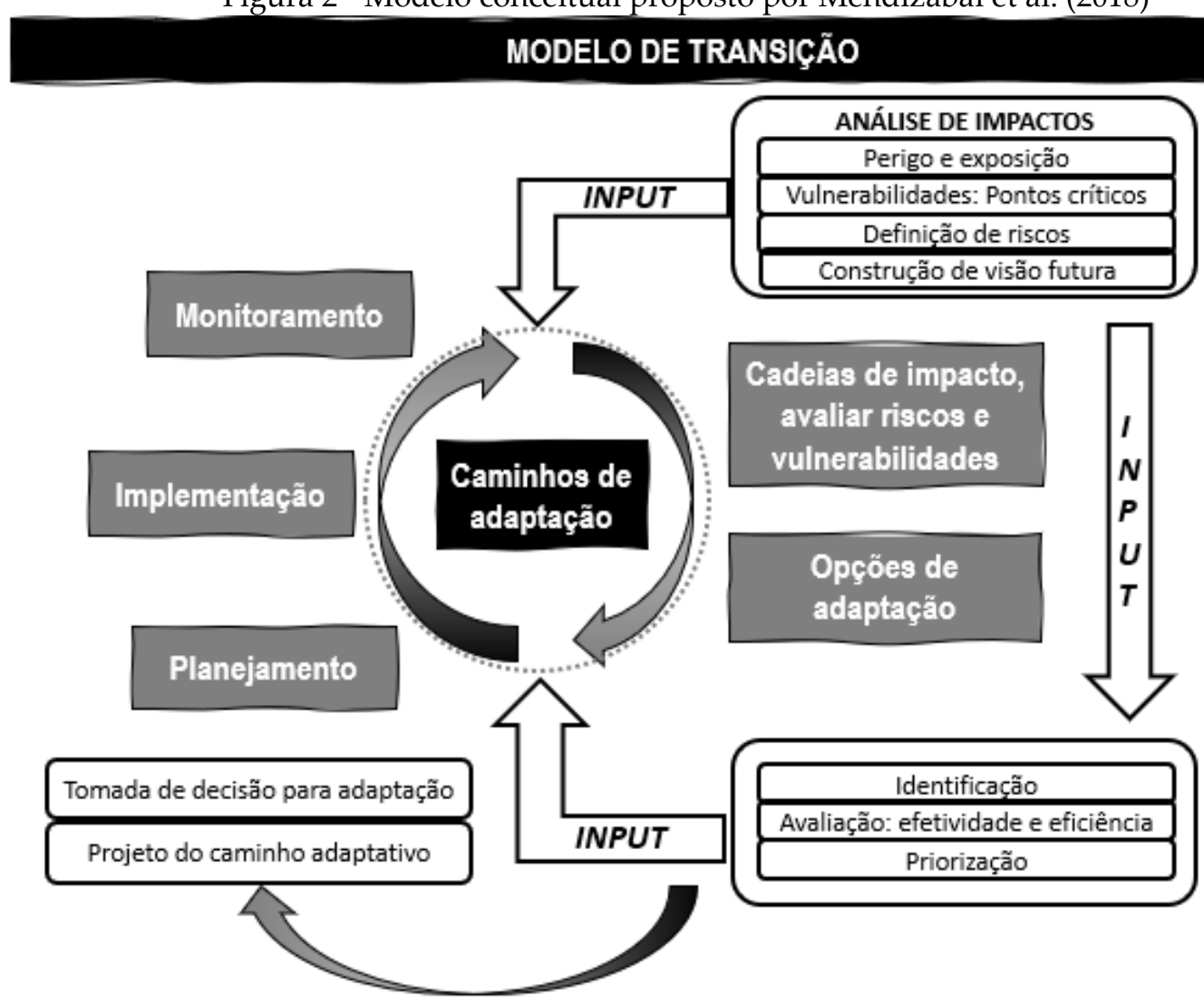

Como última fase do delineamento da pesquisa, a etapa de Reflexão cobre as atividades (7) e (9) do trabalho. Compreende a implementação e posterior avaliação das versões iniciais do método de avaliação de resiliência urbana, juntamente com especialistas das áreas de planejamento urbano e de sistemas de transporte urbano.

Diante disso, sucessivos ciclos de aplicação do método serão necessários, até que se estabeleça a sua versão final. Cabe ainda ressaltar que, apesar de o método ser implementado e avaliado no contexto urbano de Porto Alegre, entende-se que, ao promover a adaptação do modelo conceitual de Mendizabal et al. (2018) para uma capital brasileira, seus achados possibilitarão a utilização do método para outras localidades.

\section{RESULTADOS ESPERADOS E IMPLICAÇÕES DA PESQUISA}

Considerando a etapa de compreensão do método, a pesquisa está inicialmente reunindo informações, através de diferentes fontes, para identificar os atributos inerentes de sistemas de transportes que possuem maior relevância na construção de resiliência urbana. A seguir, este levantamento de informações, aliado a documentos de IFM, irá proporcionar base teórica para identificação de oportunidades de melhorias nos requisitos dessas instituições para fomentar projetos de transporte urbano que tenham impacto positivo na sustentabilidade e na resiliência.

Todas as informações coletadas ao longo das etapas descritivas do método de pesquisa irão permitir a elaboração de uma primeira versão de um método de avaliação de resiliência urbana para implementação 
e retrofit de sistemas sustentáveis de transporte em cidades. A versão final deste método de avaliação, o qual se caracteriza como o principal resultado esperado do trabalho, será baseada em ferramentas estatísticas, coleta de dados e múltiplas rodadas de aplicação com stakeholders de planejamento urbano e do sistema de transportes. Além disso, espera-se que o método desenvolvido seja válido para ser usado em cidades de diferentes contextos urbanos, como intuito de guiar investimentos direcionados à infraestrutura de transportes.

\section{REFERÊNCIAS}

AHERN, J. From fail-safe to safe-to-fail: Sustainability and resilience in the new urban world. Landscape and Urban Planning, v. 100, n. 4, p. 341-343, 30 abr. 2011.

BONILLA, M., ZAPPAROLI, I. The Challenge of Financing Urban Infrastructure for Sustainable Cities. Washington, DC: Inter-American Development Bank. 2017.

CARVALHO, C. H. Emissões relativas de poluentes do transporte urbano. Boletim regional, urbano e ambiental - Instituto de Pesquisa Econômica. 2011

COLLIER, M. J. et al. Transitioning to resilience and sustainability in urban communities. Cities, v. 32, jul. 2013.

ELMQVIST, T. et al. Sustainability and resilience for transformation in the urban century. Nature Sustainability, v. 2, n. 4, p. 267-273, 1 abr. 2019.

GOLDBECK, N.; ANGELOUDIS, P.; OCHIENG, W. Y. Resilience assessment for interdependent urban infrastructure systems using dynamic network flow models. Reliability Engineering and System Safety, v. 188, p. 62-79, 2019.

ICLEI. Resilient cities, thriving cities: The evolution of urban resilience. Bonn, Germany. 2019.

MEEROW, S.; NEWELL, J. P.; STULTS, M. Defining urban resilience: a review. Landscape and Urban Planning. Elsevier B.V., 1 mar. 2016.

MENDIZABAL, M. et al. Stimulating urban transition and transformation to achieve sustainable and resilient cities. Renewable and Sustainable Energy Reviews. Elsevier Ltd, 1 out. 2018.

PANDIT, A. et al. Infrastructure ecology: an evolving paradigm for sustainable urban development. Journal of Cleaner Production, v. 163, p. S19-S27, 1 out. 2017.

ROCKEFELLER FOUNDATION, ARUP. City resilience framework, Ove Arup \& Partners. International Limited 2014.

TIMON, M. The rise of resilience: Linking resilience and sustainability in city planning. Urban ecology at The New School in New York City, New York, 2014.

UNITED NATIONS. World Urbanization Prospects: The 2014 Revision. New York, NY: United Nations, Department of Economic and Social Affairs. 2014. 
UN HABITAT. Urbanization and Development: Emerging Futures. In World Cities Report. Nairobi, Kenya: UN Habitat. 2016. 